\title{
Communication
}

\section{Additional Solvent Ionizing Power Values for Binary Water- 1,1,1,3,3,3-Hexafluoro-2-propanol Solvents}

\author{
Dennis N. Kevill ${ }^{1, *}$ and Zoon Ha Ryu ${ }^{2}$ \\ 1 Department of Chemistry and Biochemistry, Northern Illinois University, Dekalb, Illinois 60115- \\ 2862, U.S.A.
}

2 Department of Chemistry, Dong-Eui University, 995 Eomgwangno, Busan-Jin-Gu, Busan 614-714, Korea

* Author to whom correspondence should be addressed. E-Mail: dkevill@niu.edu

Received: 19 October 2006 / Accepted: 27 October 2006 / Published: 30 October 2006

\begin{abstract}
Determinations of the specific rates of solvolysis of 1-adamantyl bromide and 1adamantyl iodide in 1,1,1,3,3,3-hexafluoro-2-propanol-water mixtures, in conjunction with earlier reported values in $80 \%$ ethanol, have led to additional $\mathrm{YBr}$ and YI solvent ionizing power values. These new values will be especially important in avoiding multicollinearity when the extended Grunwald-Winstein equation (extended by addition of a term involving solvent nucleophilicity) is used to correlate solvent-induced changes in the specific rates of solvolyses involving a bromide or iodide ion leaving group.
\end{abstract}

Keywords: 1-adamantyl bromide, 1-adamantyl iodide, 1,1,1,3,3,3-hexafluoro-2-propanol, Grunwald-Winstein equation, solvent ionizing power.

\section{Introduction}

The one-term Grunwald-Winstein equation (eqn 1) [1] was developed to correlate the specific rates of solvolytic displacements proceeding by a unimolecular ionization pathway $\left(\mathrm{S}_{\mathrm{N}} 1+\mathrm{E} 1\right.$ reactions).

$$
\log (k / k \mathrm{o})=m Y+c
$$

In eqn $1, k$ and $k$ o represent the specific rates of solvolysis of a substrate in a given solvent and in the standard solvent ( $80 \%$ ethanol- $20 \%$ water v/v), $Y$ is a scale of solvent ionizing power, $m$ is the 
sensitivity towards changes in the solvent ionizing power value and $c$ is a (usually small) constant (residual) term. The original $Y$ scale was developed using tert-butyl chloride as the standard substrate ( $m$ value set at unity).

For the correlation of bimolecular solvolytic displacements, where the solvent also functions as a nucleophile in the rate-determining step; it was proposed that a second variable should be added (eqn 2) [2], where $N$ is a measure of solvent nucleophilicity and $l$ is the sensitivity towards changes in its value.

$$
\log (k / k o)=l N+m Y+c
$$

Only in 1976 did an extensive listing of $N$ values (the $N_{\text {OTS }}$ scale) become available [3], based on the specific rates of solvolysis of methyl p-toluenesulfonate (tosylate). More recently, an $N_{\mathrm{T}}$ scale based on the solvolyses of the $S$-methyldibenzothiophenium ion has been developed [4, 5].

A further development as regards solvent ionizing power scales involved the recognition that the $Y$ scale based on tert-butyl chloride solvolyses included a necleophilic component [6-9], which could be avoided by the use of the bridgehead 1-adamantyl chloride [6] as the standard substrate. Further, the solvent ionizing power scale was found to be leaving-group dependent and scales have been independently determined for several leaving-groups(X) and termed, in general, $Y_{\mathrm{X}}$ scales [10]. These have been determined, for relatively poor leaving groups using 1-adamantyl derivatives (1) and for relatively good leaving groups using 2-adamantyl derivatives (2).

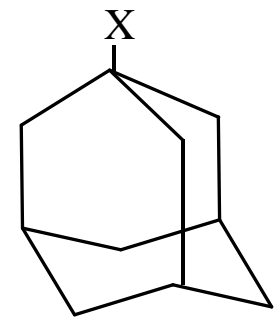

(1)

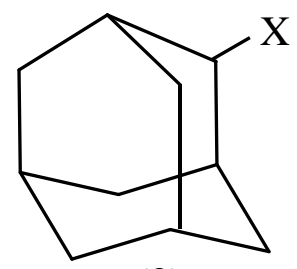

(2)

The application of eqn 2 is usually with the incorporation of the $N_{\mathrm{T}}$ scale of solvent nucleophilicity and the appropriate $Y_{\mathrm{X}}$ scale for the leaving group $\mathrm{X}$.

An important aspect of the use of eqn. 2 is that, for the majority of the binary solvent mixtures used as the solvents, as the $Y_{\mathrm{X}}$-value increases on increasing the water content, the $N_{\mathrm{T}}$ value decreases approximately linearly and multicollinearity can be a major problem. Indeed, if, as historically was often the case, a study of the solvolyses of a substrate is restricted to ethanol-water, methanol-water, acetone-water, and dioxane-water mixtures, multicollinearity is unavoidable [11, 12]. Fortunately multicollinearity problems can be avoided by the incorporation of fluoroalcohol-containing solvents and 2,2,2-trifluoroethanol (TFE)-water and 1,1,1,3,3,3-hexafluoro-2-propanol (HFIP)-water mixtures are strongly recommended systems for incorporation into any study involving the application of eqn. 2 [5, 10]. TFE-ethanol mixtures are also frequently used [13]. The fluoroalcohol-containing binary systems make an essential contribution because of the quite different relationships between $N_{\mathrm{T}}$ and $Y_{\mathrm{X}}$ values relative to those for the other four mixed solvent systems mentioned above. For example, in TFE- $\mathrm{H}_{2} \mathrm{O}$ mixtures, as $N_{\mathrm{T}}$ increases the $Y_{\mathrm{X}}$ values show a modest increase [5,10].

Fewer $Y_{\mathrm{X}}$ values are available for $\mathrm{HFIP}-\mathrm{H}_{2} \mathrm{O}$ mixtures and the review by Bentley and Llewellyn lists a $Y_{\mathrm{X}}$ value for the important chloride, bromide, and iodide leaving groups only for a $97 \%$ HFIP (w/w) 
content. A subsequent extension of the previous [6,10] kinetic study of 1-adamantyl chloride solvolyses has led to the availability of $Y_{\mathrm{Cl}}$ values for more aqueous $\mathrm{HFIP}-\mathrm{H}_{2} \mathrm{O}$ mixtures [8]. In the present study, we determine the specific rates of solvolysis of 1-adamantyl bromide and 1-adamantyl iodide in these more aqueous mixtures, which allows the determination of the corresponding $Y_{\mathrm{Br}}$ and $Y_{\mathrm{I}}$ values.

\section{Results and Discussion}

The specific rates of solvolyses were determined, at $25.0^{\circ} \mathrm{C}$, for 1 -adamantyl bromide and 1adamantyl iodide in 97\%, 90\%, 70\%, and 50\% HFIP (weight-weight basis, with other component water). The values (Table 1) for 97\% HFIP are in very good agreement with earlier determinations $[14,15]$. The specific rates are the averages from at least four independent kinetic runs. The specific rates are reported within Table 1, together with the calculated $Y_{\mathrm{Br}}$ and $Y_{\mathrm{I}}$ values and, for comparison, previously reported $[6,8] Y_{\mathrm{Cl}}$ values.

Table 1. Specific rates of solvolyses of 1 -adamantyl halides ${ }^{a}$ in $80 \%$ ethanol and $\mathrm{HFIP}-\mathrm{H}_{2} \mathrm{O}$ mixtures at $25.0^{\circ} \mathrm{C}$ and new solvent ionizing power values $\left(Y_{\mathrm{X}}\right)$.

\begin{tabular}{|c|c|c|c|c|c|c|c|}
\hline \multirow[b]{2}{*}{ solvent $^{b}$} & \multicolumn{3}{|l|}{$1-\mathrm{AdBr}^{c}$} & \multicolumn{3}{|l|}{$1-\mathrm{AdI}^{d}$} & \multirow{2}{*}{$\frac{1-\mathrm{AdCl}}{Y_{\mathrm{Cl}}^{f}}$} \\
\hline & rate constant, $\mathrm{s}^{-1}$ & $\mathrm{~N}^{e}$ & $\mathrm{Y}_{\mathrm{Br}}$ & rate constant, $\mathrm{s}^{-1}$ & $\mathrm{~N}^{e}$ & $\mathrm{Y}_{\mathrm{I}}$ & \\
\hline $80 \mathrm{EtOH}$ & $2.8 \times 10^{-7 g}$ & & 0.00 & $5.8 \times 10^{-7 h}$ & & 0.00 & 0.00 \\
\hline \multirow{3}{*}{ 97HFIP } & $(9.1 \pm 0.2) \times 10^{-3 i}$ & & $4.51^{g}$ & $(4.00 \pm 0.02) \times 10^{-3 h}$ & & $3.84^{h}$ & $5.08^{g}$ \\
\hline & $(9.68 \pm 0.01) \times 10^{-3 h}$ & & 4.54 & - & & & \\
\hline & $(9.37 \pm 0.06) \times 10^{-3}$ & 4 & 4.52 & $(4.27 \pm 0.06) \times 10^{-3}$ & 4 & 3.87 & 5.17 \\
\hline 90HFIP & $(2.29 \pm 0.05) \times 10^{-3}$ & 5 & 3.91 & $(1.63 \pm 0.03) \times 10^{-3}$ & 4 & 3.45 & 4.31 \\
\hline 70HFIP & $(1.09 \pm 0.04) \times 10^{-3}$ & 4 & 3.59 & $(1.24 \pm 0.07) \times 10^{-3}$ & 4 & 3.33 & 3.83 \\
\hline 50HFIP & $(1.06 \pm 0.04) \times 10^{-3}$ & 4 & 3.58 & $(1.25 \pm 0.05) \times 10^{-3}$ & 4 & 3.33 & 3.80 \\
\hline
\end{tabular}

${ }^{a}$ With associated standard errors. ${ }^{b} 80 \mathrm{EtOH}$ on volume-volume basis and HFIP-containing solvents on weight-weight basis, other component is $\mathrm{H}_{2} \mathrm{O} .{ }^{c}$ Concentration of $8.1 \times 10^{-4} \mathrm{M}$. ${ }^{d}$ Concentration of $6.6 \times 10^{-4} \mathrm{M} .{ }^{e}$ Number of determinations. ${ }^{f}$ From ref. $8 .{ }^{g}$ Data from ref. $6 .{ }^{h}$ Data from ref. 14. ${ }^{i}$ Data from ref. 15.

Differences in $Y_{\mathrm{X}}$ values for a given solvent are believed to be primarily due to differences in electrophilic solvent assistance [6] and the halide series, showing a reduction in electrophilicity as one moves down the periodic table, is a useful one for assessing the significance of the specific anion solvation [14].

It was found [14] that, while 1-adamantyl iodide reacts in most solvents faster than 1-adamantyl bromide, the rates become essentially identical in 97\% TFE and the order was reversed in the most acidic solvents studied: 97\% HFIP, acetic acid, and formic acid. In the present study (Table 1), we find the bromide to be the more reactive in both 97\% HFIP and 90\% HFIP. The rates would become equal at about $77 \%$ HFIP and the iodide is $14 \%$ and $18 \%$ more reactive in $70 \%$ and $50 \%$ HFIP. Clearly, there is a balance between the resistance of the carbon-halogen bond towards heterolysis and the magnitude of the superimposed electrophilic assistance from the solvent. 
When the comparison is made in terms of $Y_{\mathrm{x}}$ values, it is important to realize that, for a given solvent, the $Y_{\mathrm{x}}$ values are relative to the behavior of that particular 1-AdX substrate in $80 \%$ ethanol. Variations in $Y_{\mathrm{x}}$ values for different leaving groups will have components both from differences in interactions in the solvent under consideration and from differences in interactions in the $80 \%$ ethanol. However, the observation that the 1-AdI/1-AdBr specific rate ratio varies only from 2.3 in $90 \% \mathrm{EtOH}$ to 1.9 in $60 \% \mathrm{EtOH}$ [14] suggests that interactions in ethanol-rich ethanol-water mixtures are similar for different halides. In contrast, appreciably larger variation (0.46 to 1.14) is observed over the similar concentration range of $97 \%$ HFIP to $70 \%$ HFIP, consistent with the dominant influence of differences in the interactions of the various 1-adamantyl halides with a more electrophilic fluoroalcohol-rich solvent.

In addition to the obvious utility in correlations using eqn 1, the presently reported $Y_{\mathrm{Br}}$ and $Y_{\mathrm{I}}$ values for HFIP- $\mathrm{H}_{2} \mathrm{O}$ mixtures will be especially useful in extended Grunwald-Winstein equation (eqn 2) treatments of solvolyses involving displacement of bromide or iodide ion. In conjunction with available $N_{\mathrm{T}}$ values for the solvents [4,5], they will assist in promoting a good mix of solvent types. This will avoid the high degree of multicollinearity present when only specific rates determined in binary mixtures of water with acetone, dioxane, ethanol, and methanol are available.

\section{Experimental}

The 1-adamantyl bromide (Aldrich, 99\%) and 1-adamantyl iodide (Aldrich, 98\%) were used as received. The 1,1,1,3,3,3-hexafluoro-2-propanol (Aldrich, 99+\%) was purified as previously described [15]. The apparatus, allowing rapid response to changes in conductivity [16], has been previously described [17]. Typically, the determinations of the specific rates were made after the injection of 4.0 $\mu \mathrm{l}$ of a $10 \%$ (weight-weight) solution of the substrate in dry acetonitrile into the conductivity cell containing $2.00 \mathrm{~mL}$ of the solvent mixture.

\section{Acknowledgements}

Acknowledgment is made to the donors of the American Chemical Society Petroleum Research Fund for partial support of this research. This work was also supported by Dong-Eui University (sabbatical leave to Z.H.R. in the 2005 research year). D.N.K. thanks Prof. H. Mayr, Universität München for hospitality during the time that this manuscript was being prepared.

\section{References and Notes}

1. Grunwald, E.; Winstein, S. The Correlation of Solvolysis Rates. J. Am. Chem. Soc. 1948, 70, 846.

2. Winstein, S.; Grunwald, E.; Jones, H.W. The Correlation of Solvolysis Rates and the Classification of Solvolysis Reactions into Mechanistic Categories. J. Am. Chem.Soc. 1951, 73, 2700.

3. Schadt, F.L.; Bentley, T.W.; Schleyer, P.v.R. The $\mathrm{S}_{\mathrm{N}} 2-\mathrm{S}_{\mathrm{N}} 1$ Spectrum.2. Quantitative Treatments of Nucleophilic Solvent Assistance. A Scale of Solvent Nucleophilicities. J. Am. Chem. Soc. 1976, 98, 7667.

4. Kevill, D.N.; Anderson, S.W. An Improved Scale of Solvent Nucleophilicity Based on the Solvolysis of the S-Methyldibenzothiophenium Ion. J. Org. Chem. 1991, 56, 1845. 
5. Kevill, D.N. Development and Uses of Scales of Solvent Nucleophilicity. In Advances in Quantitative Structure-Property Relationships, Vol 1; Charton, M., Ed.; JAI Press: Greenwich, CT, 1996; pp 81-115.

6. Bentley, T.W.; Carter G.E. The $\mathrm{S}_{\mathrm{N}} 2-\mathrm{S}_{\mathrm{N}} 1$ Spectrum. 4. Mechanism for Solvolyses of tert-Butyl Chloride: A Revised $Y$ Scale of Solvent Ionizing Power based on Solvolyses of 1-Adamantyl Chloride. J. Am. Chem. Soc. 1982, 104, 5741.

7. (a) Bentley, T.W.; Bowen, C.T.; Parker, W.; Watt, C.I.F. $\mathrm{S}_{\mathrm{N}} 2$ Character of Solvolyses of tert-Butyl Halides and of Trifluoroacetolyses of Secondary Alkyl Sulfonates. J. Am. Chem. Soc. 1979, 101, 2486. (b) Bentley, T.W.; Roberts, K. Weakly Nucleophilic Leaving Groups. Solvolyses of 1Adamantyl and $t$-Bu Heptafluorobutyrates and Trifluoroacetates. J. Chem. Soc., Perkin Trans. 2 1989, 1055.

8. Kevill, D.N.; D’Souza, M.J. Additional $Y_{\mathrm{Cl}}$ Values and the Correlation of the Specific Rates of Solvolysis of tert-Butyl Chloride in Terms of $N_{\mathrm{T}}$ and $Y_{\mathrm{Cl}}$ Scales. J. Chem. Res. Synop. 1993, 174.

9. Kevill, D.N.; Anderson, S.W.; Fujimoto, E.K. Nucleophilicity Studies of Reactions in Which the Displaced Group is a Neutral Molecule. In Nucleophilicity; Harris, J.M.; McManus, S.P., Eds.; Advances in Chemistry Series, No. 215, American Chemical Society: Washington, DC, 1987; pp 269-283.

10. Bentley, T.W.; Llewellyn, G. Yx Scales of Solvent Ionizing Power. Prog. Phys. Org. Chem. 1990, $17,121$.

11. Kevill, D.N.; Park, B.-C.; Park, K.-H.; D’Souza, M.J.; Yaakoubd, L.; Mlynarski, S.L.; Kyong, J.B. Rate and Product Studies in the Solvolyses of $N, N$-Dimethylsulfamoyl and 2-Propanesulfonyl Chlorides. Org. Biomol. Chem. 2006, 4, 1580.

12. Kaspi, J.; Rappoport, Z. Nucleophilicity and Ionizing Power in Binary Solvent Mixtures. Tetrahedron Lett. 1977, 2035.

13. Kaspi, J.; Rappoport, Z. Solvolysis in 2,2,2-Trifluoroethanol-Water and 2,2,2-TrifluoroethanolEthanol Mixtures. Selectivity of the Intermediates and $N$ Values. J. Am. Chem. Soc. 1980, 102, 3829.

14. Bentley, T.W.; Carter, G.E.; Roberts, K. Solvent Ionizing Power. Comparisons of Solvolyses of 1Adamantyl Chlorides, Bromides, Iodides, and Tosylates in Protic Solvents. J. Org. Chem. 1984, 49, 5183.

15. Bentley, T.W.; Bowen, C.T.; Parker, W.; Watt, C.I.F. Evidence against Appreciable Internal Ion Pair Return in the Solvolyses of Tertiary Aliphatic Halides. Measurement of $\alpha$-Methyl/Hydrogen Rate Ratios in Hexafluoropropan-2-ol-Water. J. Chem. Soc., Perkin Trans. 2 1980, 1244.

16. Bentley, T.W.; Jones, R.O. Stoichiometric Solvation Effects. Part 1. New Equations Relating Product Selectivities to Alcohol-Water Solvent Compositions for Hydrolyses of $p$-Nitrobenzoyl Chloride. J. Chem. Soc., Perkin Trans. 2 1993, 2351.

17. Lee, I.; Lee, H.W.; Uhm, T.S.; Sung, D.D.; Ryu, Z.H. Solvation in Mixed Solvents (VII). Solvolysis of tert-Butyl Halide in Isodielectric Solvents. J. Korean Chem. Soc. 1988, 32, 85

(C) 2006 by MDPI (http://www.mdpi.org). Reproduction is permitted for noncommercial purposes. 